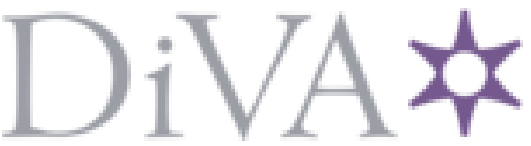

http://www.diva-portal.org

Postprint

This is the accepted version of a paper presented at Power Systems Computation Conference (PSCC) 2018.

Citation for the original published paper:

Herre, L., Söder, L., Mathieu, J L. (2018)

The Flexibility of Thermostatically ControlledLoads as a Function of Price Notice Time

In: Dublin

N.B. When citing this work, cite the original published paper.

Permanent link to this version:

http://urn.kb.se/resolve?urn=urn:nbn:se:kth:diva-231946 


\section{The Flexibility of Thermostatically Controlled Loads as a Function of Price Notice Time}

\author{
Lars Herre, Lennart Söder \\ Department of Electric Power \& Energy Systems \\ KTH Royal Institute of Technology \\ 10044 Stockholm, Sweden \\ \{lherre, lsod\}@kth.se
}

\author{
Johanna L. Mathieu \\ Department of Electrical Engineering \& Computer Science \\ University of Michigan \\ Ann Arbor, MI 48109 USA \\ jlmath@umich.edu
}

\begin{abstract}
Due to increased use of variable renewable energy sources, more capacity for balancing and ancillary services (AS) is required. Non-generating resources such as thermostatically controlled loads (TCLs) can arbitrage energy prices and provide AS due to their thermal energy storage capacity. This paper explores the impact of energy/AS price notice time, i.e. the time between when the price is announced and when it takes effect, on the TCL energy consumption and AS capacity bids, and quantifies trade-offs between notice time and flexibility. We first optimize the energy consumption and AS capacity offers at a given notice time, varied from 24 hours ahead to real-time. We then introduce uncertainty in TCL availability, formulate the stochastic optimization problem, and evaluate how the trade-offs change. We find that price notice time impacts TCL profits, but does not significantly affect the total AS capacity offered over the day. However, AS capacity offers are impacted by uncertainty, which is likely to increase with notice time.
\end{abstract}

Index Terms-ancillary services, demand response, electricity prices, notice time, thermostatically controlled loads

\section{INTRODUCTION}

An increasing use of variable renewable energy sources requires more flexible resources that can respond in realtime to supply/demand imbalance. To this end, thermostatically controlled loads (TCLs) have been proposed for energy arbitrage [1] and ancillary services [2]-[6]. Residential TCLs such as air conditioners, heat pumps, water heaters, and refrigerators, represent about $20 \%$ of the total electricity consumption in the United States [7], and thus present a large resource for providing various balancing and ancillary services (AS) to the grid. By exploiting the inherent thermal energy storage capacity of TCLs, their electricity consumption can be varied while still meeting the desired temperature range of the end user.

The cost-optimal power consumption schedule of a TCL population depends on electricity prices. The cost-optimal power capacity offered to the ancillary services (AS) market depends on AS prices and the time for which actions need to be sustained. Both depend on ambient temperatures, which affects TCL operation [8] and availability (e.g., air conditioners are only available for scheduling/AS if it is sufficiently hot outside and they are powered on), and price notice time, which is the time between when the price is announced and when it takes effect. Furthermore, the energy consumption schedule impacts the feasible AS market capacity offering and so the problems must be solved together.

In this paper, we study the impact of price notice time on the a TCL aggregator's profits and the flexibility offered to the system when TCL aggregations both arbitrage energy prices and provide AS. We first formulate a deterministic optimization problem that maximizes aggregator profit subject to prices that arrive with notice times of 0 to 24 hours and assuming that ambient temperature forecasts are perfect. We then reformulate the problem as a stochastic optimization problem wherein temperature forecasts are imperfect, affecting TCL availability. We formulate the stochastic optimization problem using chance constraints. Since we are interested in developing a qualitative understanding of the relationship between profits/flexibility and price notice time, we use a simplified thermal energy storage model of the dynamics of TCL aggregations [1].

The main contributions of this paper are the formulation of the aggregator's profit maximization problem for both the deterministic and stochastic case and case study results demonstrating the impact of notice time and stochasticity on aggregator profits and AS capacity offers. We show that profits increase with increased notice time up to a point, and then stay constant. We also show that notice time does not have a strong affect on TCL flexibility but stochasticity negatively impacts profits and TCL flexibility.

The rest of the paper is structured as follows. In Section II we describe the problem setup. The deterministic and stochastic formulations are given in Section III. The results of a case study with ISO New England market data is presented in Section IV and Section V concludes.

\section{Problem Setup}

\section{A. Thermal Energy Storage Model of a TCL Aggregation}

An aggregation of TCLs can be modeled as a thermal energy storage device capable of shifting energy usage in time due to the thermal energy storage in buildings and appliances [1], [6]. We use the model from [1], which captures the evolution of the energy state $S_{t}$ at the end of time $t$ (referred to here as its state of charge (SOC)) with respect to the power consumption $P_{t}^{E n}$ :

$$
S_{t}=S_{t-1}+\Delta\left(P_{t}^{E n}-B_{t}\right) .
$$


where $B_{t}$ is the baseline power consumption, which is the consumption required to keep $S_{t}$ at the same level, and $\Delta$ is the time step. Aggregations of TCLs have both energy and power capacity constraints, i.e., $\underline{P}_{t} \leq P_{t}^{E n} \leq \bar{P}_{t}$ and $\underline{S}_{t} \leq S_{t} \leq \bar{S}_{t}$, which ensure that there is no decrease in service to the electricity customers. In this work, we consider a population of 1000 air conditioning units with heterogenous thermal parameters, temperature set points and temperature dead-bands. In [1], the parameters $Y_{t}=\left[B_{t}, \bar{P}_{t}, \underline{P}_{t}, \bar{S}_{t}, \underline{S}_{t}\right]$ are defined analytically as a function of the individual TCL parameters and ambient temperature. We use the same parameters and method as [1] to calculate $Y_{t}$, obtaining the same thermal energy storage model parameters (as a function of outdoor air temperature) as shown in Fig. 2 of [1] (analytically computed parameters). As shown in [9], the model is imperfect; it generally overestimates the capacity of the resource. However, it allows us to obtain qualitatively accurate trends without the computational challenges posed by a more detailed model.

\section{B. Assumptions about the markets}

In this section, we outline the two market formats and their relevant quantities.

- The Energy Market (EM) accepts price-quantity bids until gate closure and energy prices are published for each market interval $\Delta^{E M}$ with a notice time $T_{n}^{E M}$ before the market interval starts.

- The Ancillary Services Market (AM) accepts bids for reserve capacity until gate closure and releases the AS prices for each market interval $\Delta^{A M}$ at a notice time $T_{n}^{A M}$ before the market interval starts. All accepted bids in the AM are paid for their capacity, independent of whether the reserves are activated. In the event of noncompliance with the AS signal, a penalty is due. In this paper, we assume that the TCL aggregator plans to comply, i.e., the penalty is not explicitly considered in the optimization problem.

Here, we use $\Delta=\Delta^{E M}=\Delta^{A M}=5 \mathrm{~min}$, which is in line with the market setup of ISO New England [10], and $T_{n}=T_{n}^{E M}=T_{n}^{A M}$, where we vary $T_{n}$ from 0 to 24 hours.

Market participants are the TCL aggregator, as well as other aggregators and generators. While their upfront costs may be non-trivial, TCLs have low marginal costs associated with participation in energy/AS markets (e.g., costs associated with operations/ maintenance and energy losses resulting from external control actions). Therefore, we assume their marginal costs are zero. We also assume that they are a relatively small market participant and therefore they are price takers. Given these assumption, we can treat EM/AM prices as exogenous and so the TCL aggregator optimizes its consumption and AS bids with respect to known future prices and the ISO commits its entire AS bid.

Ignoring energy losses, energy capacity constraints require that a TCL follows a signal that is zero mean with respect to its baseline operation over a particular time interval. Independent system operators (ISOs) have developed a variety of mechanisms to ensure certain AS signals are zero mean, e.g.,

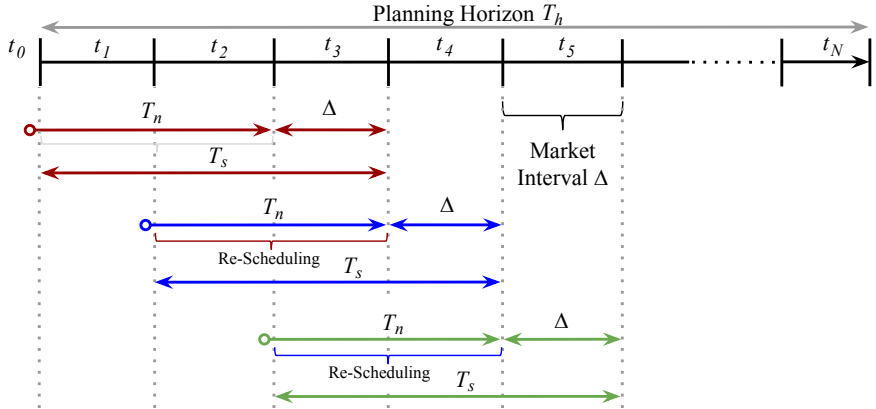

Figure 1. Example of relevant time intervals: notice time $\left(T_{n}=10 \mathrm{~min}\right)$, market interval $(\Delta=5 \mathrm{~min})$, scheduling period $\left(T_{s}=15 \mathrm{~min}\right)$, re-scheduling period $(10 \mathrm{~min})$, and planning horizon $\left(T_{h}=24 \mathrm{~h}\right)$.

California ISO's Regulation Energy Management [11] and PJM's RegD signal. In this paper we assume that AS signals are zero mean over each interval $\Delta$ and that TCL aggregators bid equivalent upwards and downwards AS capacity.

The relevant time intervals including $T_{n}$ and $\Delta$ are shown in Fig. 1. The price is constant during each $\Delta$. If $T_{n} \geq \Delta$, it becomes possible to re-schedule those market intervals that have previously been scheduled, which is beneficial if new or better information about the future is available. The total period over which power is optimized $T_{n}+\Delta$ is referred to as the scheduling period $T_{s}$. The total time frame is termed the planning horizon $T_{h}$.

\section{PROBlEM Formulation}

The objective of the aggregator is to maximize its revenue which is comprised of its profit from AS capacity $P^{A S}$ and cost from electricity consumption $P^{E n}$, where $\lambda^{A S}$ and $\lambda^{E n}$ are the AS and energy prices, respectively. The variables and parameters are summarized in Table I. We assign a cost for SOC deviations from $50 \%$ at the end of the planning horizon, and so the overall energy consumption over the entire planning horizon is approximately equal to the baseline consumption. However, the timing of the consumption and thus the available AS capacity can differ.

\section{A. Deterministic Formulation}

We first formulate the deterministic problem

$$
\begin{array}{r}
\operatorname{max.\Delta } \sum_{t=t_{1}}^{t_{N}}\left(\lambda_{t}^{A S} P_{t}^{A S}-\lambda_{t}^{E n} P_{t}^{E n}\right)-c \delta \\
\text { s.t. } S_{t}=S_{t-1}+\Delta\left(P_{t}^{E n}-B_{t}\right) \forall t \\
P_{t}^{E n}+P_{t}^{A S} \leq \bar{P}_{t} \forall t \\
P_{t}^{E n}-P_{t}^{A S} \geq \underline{P}_{t} \forall t \\
S_{t}+\frac{\Delta}{2} P_{t}^{A S} \leq \bar{S}_{t} \forall t \\
S_{t}-\frac{\Delta}{2} P_{t}^{A S} \geq \underline{S}_{t} \forall t \\
\leq S_{t_{N}} \leq G_{t_{N}}+\delta \\
\delta \geq 0 \\
S_{t_{0}}=G_{t_{0}}
\end{array}
$$


TABLE I

OPTIMIZATION PROBLEM VARIABLES AND PARAMETERS

\begin{tabular}{llr}
\hline Variable & Description & \\
\hline$P_{t}^{E n}, P_{t}^{A S}$ & Consumption and AS capacity at time $t$ & \\
$S_{t}$ & SOC at time $t$ & \\
$\delta$ & Deviation from 50\% SOC & Value \\
\hline Parameter & Description & see Fig. 6 \\
\hline$\lambda_{t}^{E n}, \lambda_{t}^{A S}$ & Energy and AS price at time $t$ & $22-33^{\circ} \mathrm{C}$ \\
$\theta_{t}$ & Ambient temperature & $f\left(\theta_{t}\right)[1]$ \\
$\bar{P}_{t}, \underline{P}_{t}$ & Bounds on $P_{t}^{E n}$ at time $t$ & $f\left(\theta_{t}\right)[1]$ \\
$\bar{S}_{t}, \underline{S}_{t}$ & Bounds on $S_{t}$ at time $t$ & $f\left(\theta_{t}\right)[1]$ \\
$B_{t}$ & Baseline power consumption at time $t$ & $1 \frac{\$}{\mathrm{kWh}}$ \\
$c$ & Cost associated to SOC deviation $\delta$ & \\
\hline
\end{tabular}

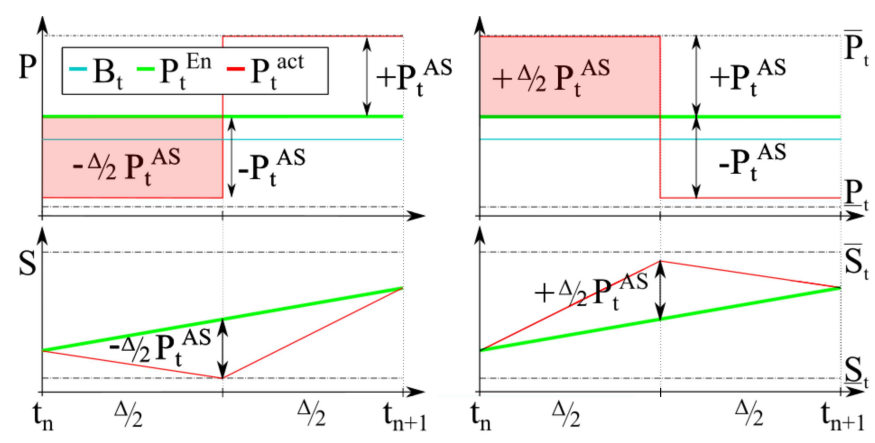

Figure 2. Examples of worst case AS signal $P_{t}^{a c t}$ (top) and associated SOC deviations (bottom). Left: First the full downwards AS capacity is activated and then the full upwards capacity is activated. The lower energy capacity constraint becomes active, before the lower power bound is hit. Right: First the full upwards AS capacity is activated and then the full downwards capacity is activated. The upper power capacity constraint becomes active before the the upper energy bound is hit.

where (2) maximizes the profit from AS capacity minus the cost for energy consumption. The last term implements the penalty associated with deviations for $50 \%$ SOC at the end of the planning horizon (otherwise the SOC will go to zero at the end of the horizon). Constraint (3) models the evolution of the SOC, while (4) and (5) constrain the consumption and AS capacity (assuming the maximum AS signal is equal to the capacity) and (6) and (7) constrain the SOC in a conservative way. Specifically, they constrain the worst-case SOC, which occurs when the actual AS signal $P_{t}^{a c t}$ is equal to $\pm P_{t}^{A S}$ during the first $\frac{\Delta}{2}$ and to $\mp P_{t}^{A S}$ during the second $\frac{\Delta}{2}$, as shown in Fig. 2. Constraint (8) defines the SOC deviation $\delta$, where $G_{t}=\frac{1}{2} \cdot\left(\bar{S}_{t}+\underline{S}_{t}\right)$ and (9) ensures $\delta$ is positive. Constraint (10) sets the initial SOC to $50 \%$.

\section{B. Stochastic Formulation}

In practice we would not know the parameters $Y_{t}=$ $\left\{B_{t}, \bar{P}_{t}, \underline{P}_{t}, \bar{S}_{t}, \underline{S}_{t}\right\}$ perfectly for the following reasons.

- Temperature. The parameters are a function of ambient temperature and temperature forecasts are inherently uncertain.

- Behavior. The model assumes that air conditioners subject to outdoor temperatures above their dead-bands are powered on and available for aggregator control. In practice, customers may choose to keep their air conditioners off even when it is warm outside.
- Model. The thermal energy storage model is imperfect because it does not capture the full dynamics, constraints, and disturbances associated with each individual TCL.

Therefore, we propose a stochastic formulation where the parameters are treated as random variables $\mathcal{Y}_{t}=$ $\left\{\mathcal{B}_{t}, \overline{\mathcal{P}}_{t}, \underline{\mathcal{P}}_{t}, \overline{\mathcal{S}}_{t}, \underline{\mathcal{S}}_{t}\right\}$. Since the aggregator must meet its AS commitment and additionally ensure that its actions do not violate the aggregations energy/power capacity constraints (which would translate into deviations of TCL temperatures outside of their dead-bands, violating the aggregator's contracts with electricity customers), we employ chance constraints which ensure that stochastic constraints hold with a probability of $\alpha_{i}$ or higher. The chance constrained problem is

$$
\begin{aligned}
& \operatorname{max.\Delta } \sum_{t=t_{1}}^{t_{N}}\left(\lambda_{t}^{A S} P_{t}^{A S}-\lambda_{t}^{E n} P_{t}^{E n}\right)-c \delta \\
& \text { s.t. } S_{t}=S_{t-1}+\Delta\left(P_{t}^{E n}-B_{t}\right) \forall t \\
& \operatorname{Pr}\left\{P_{t}^{E n}+P_{t}^{A S} \leq \overline{\mathcal{P}}_{t}\right\} \geq \alpha_{1} \forall t \\
& \operatorname{Pr}\left\{P_{t}^{E n}-P_{t}^{A S} \geq \underline{\mathcal{P}}_{t}\right\} \geq \alpha_{2} \forall t \\
& \operatorname{Pr}\left\{S_{t}+\frac{\Delta}{2} P_{t}^{A S} \leq \overline{\mathcal{K}}_{t}\right\} \geq \alpha_{3} \forall t \\
& \operatorname{Pr}\left\{S_{t}-\frac{\Delta}{2} P_{t}^{A S} \geq \underline{\mathcal{K}}_{t}\right\} \geq \alpha_{4} \forall t \\
& \\
& \quad \operatorname{Pr}\left\{S_{t_{N}}-\delta \leq \mathcal{G}_{t_{N}}\right\} \geq \alpha_{5} \forall t \\
& \\
& \operatorname{Pr}\left\{S_{t_{N}}+\delta \geq \mathcal{G}_{t_{N}}\right\} \geq \alpha_{6} \forall t
\end{aligned}
$$

(9) and (10)

where (11) and (12) are the same as in the deterministic case since (12) includes only the deterministic part of the stochastic parameter $\mathcal{B}_{t}=B_{t}+\beta_{t}$, where $\beta_{t}$ is the baseline forecast error. Now $S_{t}$ is the forecasted SOC, instead of the actual. The power capacity chance constraints (13) and (14) are defined in terms of the stochastic bounds $\overline{\mathcal{P}}_{t}, \underline{\mathcal{P}}_{t}$. The energy capacity chance constraints (15) and (16) are defined in terms of the stochastic bounds $\overline{\mathcal{K}}_{t}, \underline{\mathcal{K}}_{t}$ where

$$
\begin{aligned}
& \overline{\mathcal{K}}_{t}=\overline{\mathcal{S}}_{t}+\Delta \beta_{t}+\Delta \sum_{\tau=t_{1}}^{t-1} \beta_{\tau} \forall t, \\
& \underline{\mathcal{K}}_{t}=\underline{\mathcal{S}}_{t}+\Delta \beta_{t}+\Delta \sum_{\tau=t_{1}}^{t-1} \beta_{\tau} \forall t,
\end{aligned}
$$

since the baseline error aggregates over time. This corresponds to increasingly narrower bounds on the SOC over the planning horizon. Constraints (17) and (18) are the stochastic counterparts to (8) where $\mathcal{G}=\frac{1}{2} \cdot\left(\overline{\mathcal{S}}_{t_{N}}+\underline{\mathcal{S}}_{t_{N}}\right)$.

We reformulate the chance constraints (13) to (18) using the cumulative distribution function $(\mathrm{CDF}) \Phi$ of the stochastic parameters.

$$
\begin{aligned}
P_{t}^{E n}+P_{t}^{A S} & \leq \Phi_{\overline{\mathcal{P}}_{t}}^{-1}\left(1-\alpha_{1}\right) \forall t \\
P_{t}^{E n}-P_{t}^{A S} & \geq \Phi_{\underline{\mathcal{P}}_{t}^{-1}}^{-1}\left(\alpha_{2}\right) \quad \forall t \\
S_{t}+\frac{\Delta}{2} P_{t}^{A S} & \leq \Phi_{\overline{\mathcal{K}}_{t}}^{-1}\left(1-\alpha_{3}\right) \forall t \\
S_{t}-\frac{\Delta}{2} P_{t}^{A S} & \geq \Phi_{\underline{\mathcal{K}}_{t}}^{-1}\left(\alpha_{4}\right) \quad \forall t \\
S_{t_{N}}-\delta & \leq \Phi_{\mathcal{G}_{t_{N}}^{-1}}\left(1-\alpha_{5}\right) \\
S_{t_{N}}+\delta & \geq \Phi_{\mathcal{G}_{t_{N}}^{-1}}^{-1}\left(\alpha_{6}\right)
\end{aligned}
$$


If the uncertainty can be modeled, the CDFs can be computed offline and used as a lookup table to determine the quantities on the right side of each constraint.

Later we show that baseline error aggregation, as shown in (20) and (21), results in infeasibility after only a few market intervals when we use realistic uncertainty distributions. To mitigate this problem we propose a method to compensate the baseline error in each interval.

1) Baseline error compensation: At time step $t$, the aggregator knows the ambient temperature at $t-1$, i.e., $\theta_{t-1}$, and can use this to estimate the actual baseline $B_{t-1}^{a c t}{ }^{1}$. Then the actual baseline error $b_{t-1}^{a c t}=B_{t-1}^{a c t}-B_{t-1}$, which is a realization of $\beta_{t-1}$, can be compensated for by additional or less energy consumption in interval $t$. This prevents the baseline error from aggregating. However, the aggregator needs to reserve a power and energy margin to eliminate the baseline error. Therefore, the problem becomes

$$
\begin{array}{ll}
\max . \Delta \sum_{t=t_{1}}^{t_{N}} \lambda_{t}^{A S}\left(P_{t}^{A S}+E\left[\beta_{t-1}\right]\right)-\lambda_{t}^{E n} P_{t}^{E n}-c \delta \\
\text { s.t. } \quad S_{t}=S_{t-1}+\Delta\left(P_{t}^{E n}-B_{t}\right) \forall t \\
\operatorname{Pr}\left\{P_{t}^{E n}+P_{t}^{A S} \leq \overline{\mathcal{Q}}_{t}\right\} \geq \alpha_{1} \forall t \\
\operatorname{Pr}\left\{P_{t}^{E n}-P_{t}^{A S} \geq \underline{\mathcal{Q}}_{t}\right\} \geq \alpha_{2} \forall t \\
\operatorname{Pr}\left\{S_{t}+\frac{\Delta}{2} P_{t}^{A S} \leq \overline{\mathcal{L}}_{t}\right\} \geq \alpha_{3} \forall t \\
\operatorname{Pr}\left\{S_{t}-\frac{\Delta}{2} P_{t}^{A S} \geq \underline{\mathcal{L}}_{t}\right\} \geq \alpha_{4} \forall t
\end{array}
$$

$$
\text { (9), (10), (17) and (18) }
$$

where the objective function now includes the expected cost to buy/sell energy associated with baseline compensation. However, since $E\left[\beta_{t-1}\right]=0$, the objective function simplifies to the same as before. The SOC equation (29) also remains the same as before. The power capacity chance constraints (30) and (31) now include the power compensation, i.e.,

$$
\begin{aligned}
& \overline{\mathcal{Q}}_{t}=\overline{\mathcal{P}}_{t}-\beta_{t-1} \forall t \\
& \underline{\mathcal{Q}}_{t}=\underline{\mathcal{P}}_{t}-\beta_{t-1} \forall t .
\end{aligned}
$$

The energy capacity chance constraints (32) and (33) are less conservative because baseline error no longer aggregates, i.e.,

$$
\begin{aligned}
& \overline{\mathcal{L}}_{t}=\overline{\mathcal{S}}_{t}+\Delta \beta_{t}+\Delta \beta_{t-1} \forall t \\
& \underline{\mathcal{L}}_{t}=\underline{\mathcal{S}}_{t}+\Delta \beta_{t}+\Delta \beta_{t-1} \forall t .
\end{aligned}
$$

An illustration of (30) to (33) is shown in Fig. 3 where $P^{A S}=0$ for clarity. The figure shows the planned power $P_{t}^{E n}$ and energy level $S_{t}^{E n}$ with the baseline forecast $B_{t}$ as well as an actual baseline realization $B_{t}^{a c t}$ and the resulting energy level $S_{t}^{a c t}$. The average error in the baseline $b_{1}=B_{1}-B_{1}^{a c t}$ is compensated for in the following time step by consuming $P_{2}^{c o m p}=P_{2}^{E n}+b_{1}$ instead of $P_{2}^{E n}$. The average baseline error in the second interval $b_{2}$ would be compensated in a third interval.

\footnotetext{
${ }^{1}$ In practice our estimate of $B_{t-1}^{a c t}$ will not be perfect but we can extend the approach to account for its estimation error, given historical data.
}

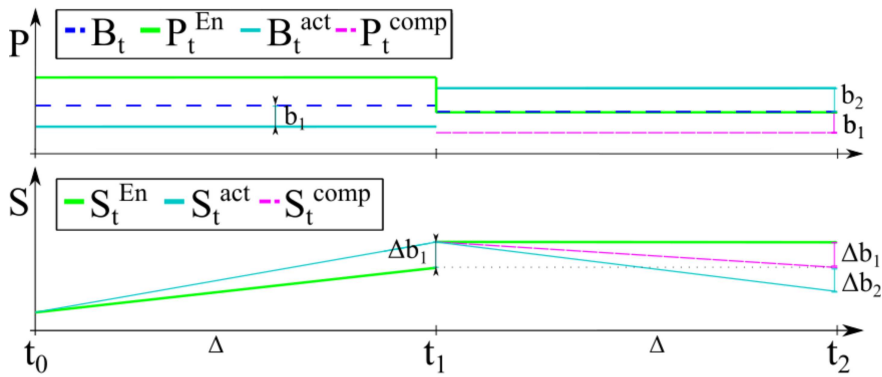

Figure 3. Correction of the average baseline error $b_{1}$ in $t_{2}$ by compensating power $P_{2}^{\operatorname{comp}}$. Here, $P^{A S}=0$ for clarity.
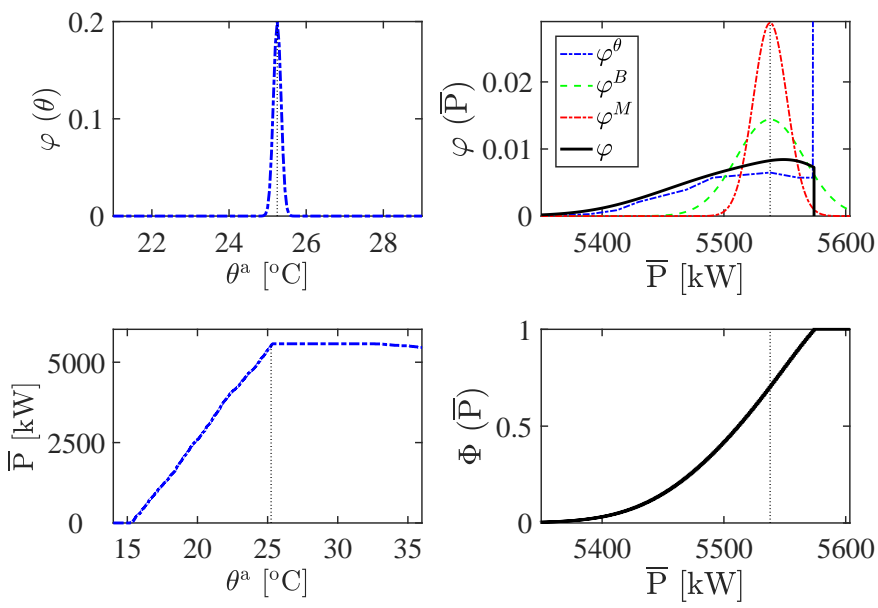

Figure 4. Method to obtain the CDF of the stochastic parameters.

We reformulate the chance constraints (30) to (33) using the CDFs of the stochastic parameters

$$
\begin{array}{ll}
P_{t}^{E n}+P_{t}^{A S} \leq \Phi_{\overline{\mathcal{Q}}_{t}}^{-1}\left(1-\alpha_{1}\right) & \forall t \\
P_{t}^{E n}-P_{t}^{A S} \geq \Phi_{\mathcal{Q}_{t}}^{-1}\left(\alpha_{2}\right) & \forall t \\
S_{t}+\frac{\Delta}{2} P_{t}^{A S} \leq \Phi_{\overline{\mathcal{L}}_{t}}^{-1}\left(1-\alpha_{3}\right) & \forall t \\
S_{t}-\frac{\Delta}{2} P_{t}^{A S} \geq \Phi_{\mathcal{\mathcal { L }}_{t}}^{-1}\left(\alpha_{4}\right) & \forall t .
\end{array}
$$

2) Probabilistic Modeling of Uncertainty: Next, we model the CDFs of the stochastic parameters. Let $\varphi(\theta)$ be the probability density function (PDF) of the temperature forecasts, where we assume temperature error is normally distributed with mean equal to the forecast and standard deviation $\sigma^{\theta}$. Let $\varphi^{B}(\cdot)$ represent the PDFs of each parameter in $\mathcal{Y}$ where parameter uncertainty is due to behavior alone. We assume the PDFs are normal distributions with mean equal to the forecast and standard deviations $\sigma^{B}(\cdot)$. Let $\varphi^{M}(\cdot)$ represent the PDFs of each parameter in $\mathcal{Y}$ where parameter uncertainty is due to model error alone. We assume the PDFs are normal distributions with mean equal to the forecast and standard deviations $\sigma^{M}(\cdot)$.

Our goal is to use the PDFs corresponding to each component of the uncertainty to obtain a CDF for each stochastic parameter. This is illustrated in Fig. 4 where we show how we compute the $\mathrm{CDF}$ of $\overline{\mathcal{P}}$. The PDF of $\overline{\mathcal{P}}$ where the parameter uncertainty is due to temperature forecast error 
alone is denoted $\varphi^{\theta}$ and computed by using $\varphi(\theta)$ (top, left) and the relationship between $\bar{P}$ and $\theta$ (bottom, left). Assuming independence of the uncertainty sources, the total $\operatorname{PDF} \varphi$ is obtained by convolution [12], i.e.,

$$
\varphi=\varphi^{\theta} * \varphi_{0}^{B} * \varphi_{0}^{M}
$$

where $\varphi_{0}^{B}, \varphi_{0}^{M}$ are the distributions of $\varphi^{B}, \varphi^{M}$ shifted to a mean of zero.

Additionally, we have truncated and normalized the PDF to preserve its shape between 0 and its physical maximum, which for $\overline{\mathcal{P}}$ corresponds to all TCLs being on.

With this method, we can compute $\varphi_{\left(\overline{\mathcal{S}}_{t}+\Delta \beta_{t}\right)}, \varphi_{\left(\underline{\mathcal{S}}_{t}+\Delta \beta_{t}\right)}$, $\varphi_{\left(\overline{\mathcal{P}}_{t}\right)}, \varphi_{\left(\underline{\mathcal{P}}_{t}\right)}, \varphi_{\left(\mathcal{G}_{t_{N}}\right)}$ and $\varphi_{\left(\Delta \beta_{t}\right)}$. With the assumption that temperature forecasts at different time intervals are statistically independent (which is not realistic and makes our results conservative), the PDFs of the stochastic bounds $\overline{\mathcal{K}}_{t}, \underline{\mathcal{K}}_{t}$ are obtained by convolution, i.e.,

$$
\begin{aligned}
\varphi_{\left(\overline{\mathcal{K}}_{t}\right)} & =\varphi_{\left(\overline{\mathcal{S}}_{t}+\Delta \beta_{t}\right)} * \varphi_{\left(\Delta \sum_{\tau=t_{1}}^{t-1} \beta_{\tau}\right)} \\
\varphi_{\left(\underline{\mathcal{K}}_{t}\right)} & =\varphi_{\left(\underline{\mathcal{S}}_{t}+\Delta \beta_{t}\right)} * \varphi_{\left(\Delta \sum_{\tau=t_{1}}^{t-1} \beta_{\tau}\right)} \\
\varphi_{\left(\Delta \sum_{\tau=t_{1}}^{t-1} \beta_{\tau}\right)} & =\varphi_{\left(\Delta \beta_{t_{1}}\right)} * \varphi_{\left(\Delta \beta_{t_{2}}\right)} * \ldots * \varphi_{\left(\Delta \beta_{t-1}\right)}
\end{aligned}
$$

Similarly, the PDFs of the stochastic bounds $\overline{\mathcal{Q}}_{t}, \underline{\mathcal{Q}}_{t}, \overline{\mathcal{L}}_{t}, \underline{\mathcal{L}}_{t}$ are

$$
\begin{aligned}
& \varphi_{\left(\overline{\mathcal{Q}}_{t}\right)}=\varphi_{\left(\overline{\mathcal{P}}_{t}\right)} * \varphi_{\left(-\Delta \beta_{t-1}\right)} \\
& \varphi_{\left(\underline{\mathcal{Q}}_{t}\right)}=\varphi_{\left(\underline{\mathcal{D}}_{t}\right)} * \varphi_{\left(-\Delta \beta_{t-1}\right)} \\
& \varphi_{\left(\overline{\mathcal{L}}_{t}\right)}=\varphi_{\left(\overline{\mathcal{S}}_{t}+\Delta \beta_{t}\right)} * \varphi_{\left(\Delta \beta_{t-1}\right)} \\
& \varphi_{\left(\underline{\mathcal{L}}_{t}\right)}=\varphi_{\left(\underline{\mathcal{S}}_{t}+\Delta \beta_{t}\right)} * \varphi_{\left(\Delta \beta_{t-1}\right)} .
\end{aligned}
$$

From here, it is straightforward to compute the CDFs.

We assume that the uncertainty increases over the scheduling period. This is modeled by scaling the standard deviations of the PDFs by a factor $k$ as follows:

- $\sigma^{\theta}=0.01^{\circ} C \cdot k$,

- $\sigma^{B}\left(\mathcal{Y}_{i}\right)=0.0005 \cdot E\left[\mathcal{Y}_{i}\right] \cdot k$,

- $\sigma^{M}\left(\mathcal{Y}_{i}\right)=0.00025 \cdot E\left[\mathcal{Y}_{i}\right] \cdot k$,

where $\mathcal{Y}_{i}$ refers a parameter in $\mathcal{Y}$ and $k$ increases linearly over the scheduling period.

Fig. 5 shows how the stochastic energy capacity limits $\overline{\mathcal{S}}_{t}, \underline{\mathcal{S}}_{t}, \overline{\mathcal{K}}_{t}, \underline{\mathcal{K}}_{t}, \overline{\mathcal{L}}_{t}, \underline{\mathcal{L}}_{t}$ evolve over time if the chance constraints are required to hold in $95 \%$ of the cases. With a price notice time of $T_{n}<5 \mathrm{~min}$, the aggregator schedules one market interval $\Delta$ at a time and the bounds follow the dashed lines. With a price notice time of $T_{n}=24 \mathrm{~h}$, the aggregator schedules the full planning horizon all at once and the bounds follow the solid lines. In this case, without baseline compensation, the problem quickly becomes infeasible.

\section{Case Studies}

We conduct case studies using prices from ISO New England (ISO NE) [10]. We consider a hot day when most consumer's air conditioners are operating and assume the outdoor temperature varies sinusoidally between 25 and $33{ }^{\circ} \mathrm{C}$. The price and outdoor temperature profiles are shown in Fig. 6.

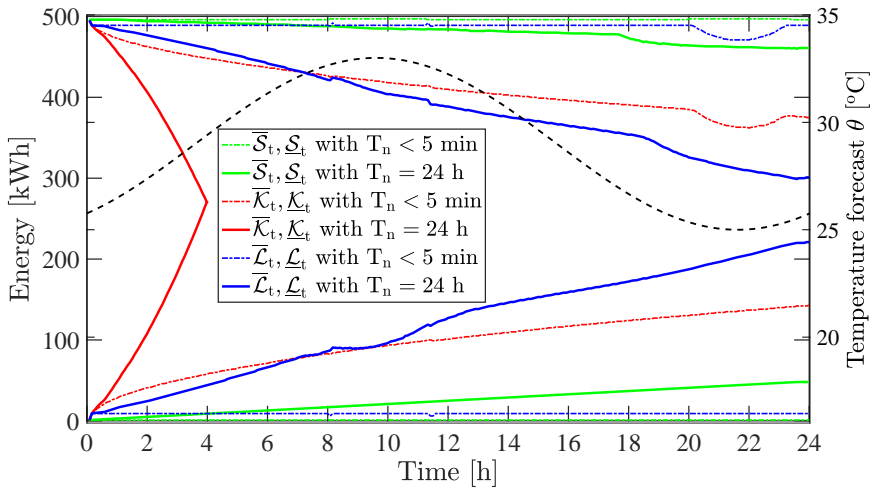

Figure 5. The chance constraint bounds of $\overline{\mathcal{S}}_{t}, \underline{\mathcal{S}}_{t}, \overline{\mathcal{K}}_{t}, \underline{\mathcal{K}}_{t}, \overline{\mathcal{L}}_{t}, \underline{\mathcal{L}}_{t}$ with varying uncertainty

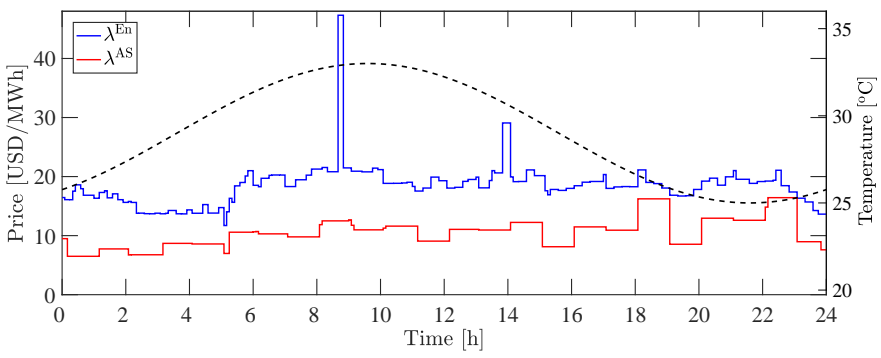

Figure 6. Price profiles from ISO NE and assumed temperature profile.

We assume that previously scheduled market intervals can be re-scheduled until right before each interval. This results in three cases:

1) $0 \leq T_{n}<5$ min: every market interval is planned separately since only a single price is known just before the start of each interval.

2) $5 \mathrm{~min} \leq T_{n}<24 \mathrm{~h}$ : The aggregator computes decisions for all market intervals for which prices are known (i.e., for the scheduling period), but only implements the decision for the upcoming interval. All future intervals are rescheduled when the next sets of prices are sent. This corresponds to model predictive control with moving horizon.

3) $T_{n}=24 \mathrm{~h}$ : The TCL aggregator computes a plan for the entire planning horizon in one shot.

First, we show a simple case with a $4 \mathrm{~h}$ planning horizon, and then we present the result of a full $24 \mathrm{~h}$ planning horizon.

\section{A. Simple Case: 4 h planning horizon}

Fig. 7 shows the results for the optimal power consumption and AS capacity bids with a notice time of $4 \mathrm{~h}$ over a $4 \mathrm{~h}$ planning horizon, i.e., when all intervals are planned together. The top figure shows the results corresponding to the deterministic formulation, and the bottom figure shows the results in corresponding to the stochastic formulation with baseline error compensation. In the deterministic case, the upper and lower power bounds are constant, whereas in the stochastic case the bounds (slightly) narrow over time due to the assumption that uncertainty increases over the scheduling horizon. 

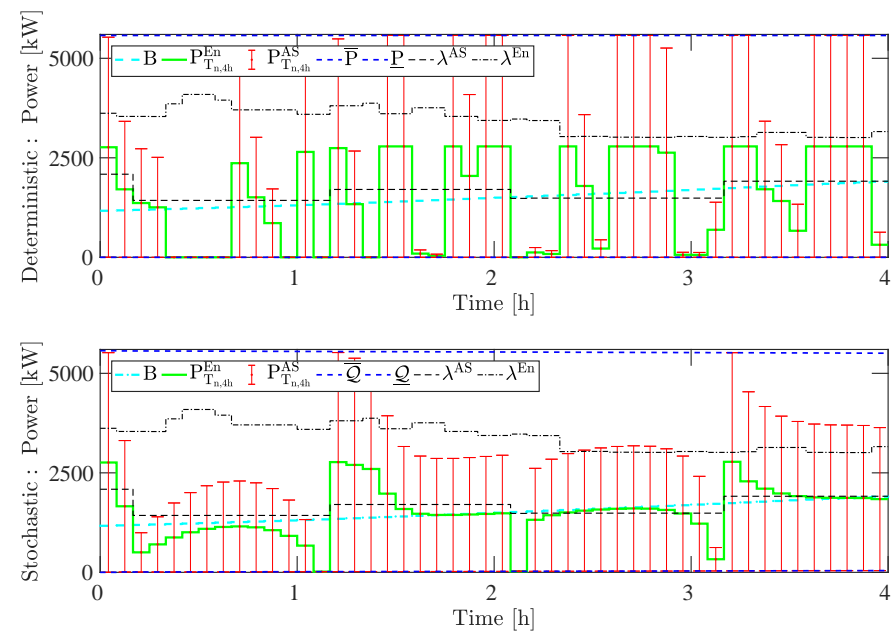

Figure 7. Time series results for the deterministic and stochastic formulations: Baseline power $B_{t}$, power consumption $P_{t}^{E n}$, AS capacity bids $P_{t}^{A S}$, and power bounds $\bar{P}_{t}, \underline{P}_{t}$ (deterministic), $\overline{\mathcal{Q}}_{t}, \underline{\mathcal{Q}}_{t}$ (stochastic) with $T_{n}=T_{h}=4$ $\mathrm{h}$. Prices are scaled to show the qualitative trajectories.
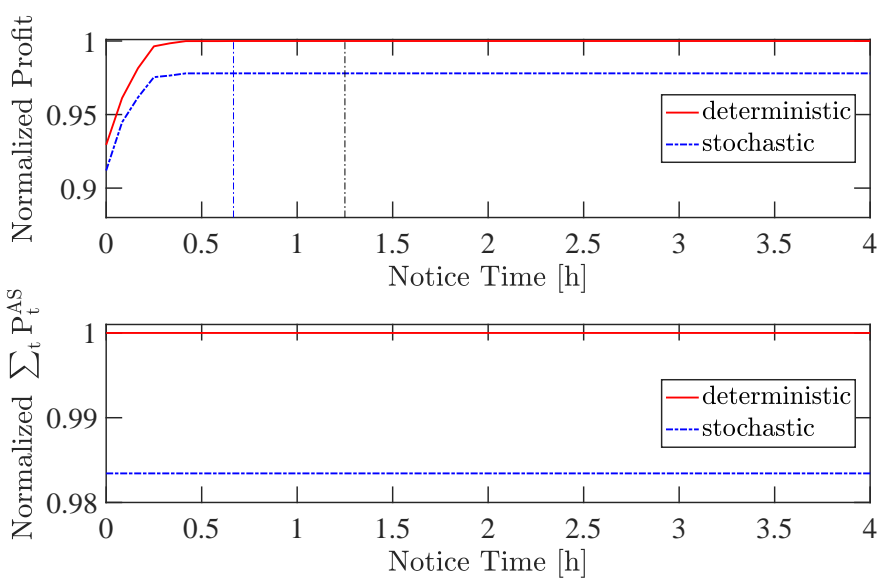

Figure 8. Comparison of normalized profits and total AS capacity over the planning horizon as a function of the notice time in the deterministic and stochastic case for a planning horizon $T_{h}=4 \mathrm{~h}$.

The results show that the energy arbitrage potentially is not fully exploited, in contrast to the simulation results in [1]. Here, the energy consumption $P_{t}^{E n}$ never exceeds $\frac{1}{2} \bar{P}_{t}$. The reason is that it is advantageous to hold back on arbitrage in order to maintain capacity for AS. Since AS capacity bids must be symmetric with respect to consumption, the largest possible AS bid corresponds to $P_{t}^{E n}=\frac{1}{2} \bar{P}_{t}$. Once the consumption exceeds that level, the AS capacity decreases since the maximum possible upward bid (which necessarily equals the downward bid) decreases.

The profits and total AS capacity over the planning horizon as a function of the notice time for the deterministic and stochastic formulations are displayed in Fig. 8. The results are normalized with respect to the maximum value of the underlying data shown in each plot. As expected, both profits and total AS capacity are larger in the deterministic case due to wider power/energy bounds. In both formulations, the profits increase with notice time, but reach a plateau after $20 \mathrm{~min}$ (deterministic) and $25 \mathrm{~min}$ (stochastic). On the other hand, the AS capacity, is not significantly impacted by notice time.

We show the normalized profit trajectory as a function of notice time in Figs. 9 and 10 for different formulations and AS and energy prices. The planning horizon is still $4 \mathrm{~h}$, though we only show the first hour since that is when the profit varies.

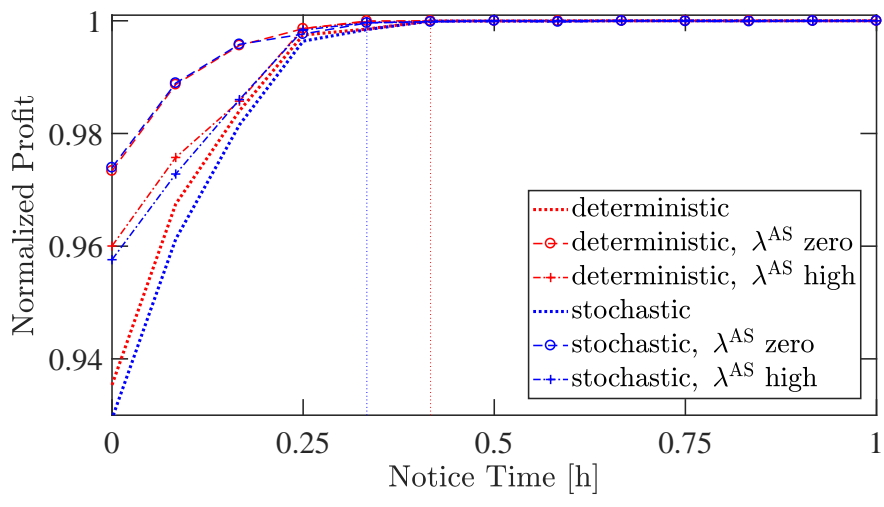

Figure 9. Normalized profits as a function of notice time for a $4 \mathrm{~h}$ planning horizon. Zero and high refer to scalings of the AS prices

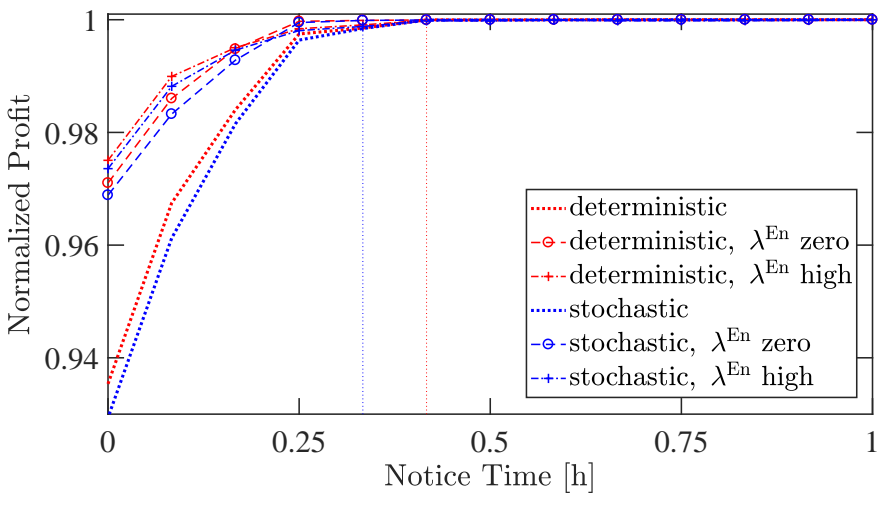

Figure 10. Normalized profits as a function of notice time for a $4 \mathrm{~h}$ planning horizon. Zero and high refer to scalings of the energy prices

Here, the profits are normalized with respect to the maximum value of each trajectory in order to compare the trajectories more closely. We find that the trajectories are qualitatively similar over different formulations and AS/energy prices.

\section{B. Full Case: 24 h planning horizon}

The results for the full $24 \mathrm{~h}$ planning horizon are shown in Fig. 11. The profits are normalized with respect to the maximum value of each trajectory. The results are qualitatively similar to the simple case. The point at which more notice time does not improve profits occurs at $T_{n}=70 \mathrm{~min}$ for the deterministic case and at $T_{n}=40 \mathrm{~min}$ for the stochastic case. Also similar to the simple case, the total AS capacity is not significantly affected by the notice time.

In our case study the AS prices are of the same order of magnitude as the energy prices affecting the optimal strategy, which is to offer as much AS capacity as possible. When we decrease the AS prices to more than one order of magnitude 


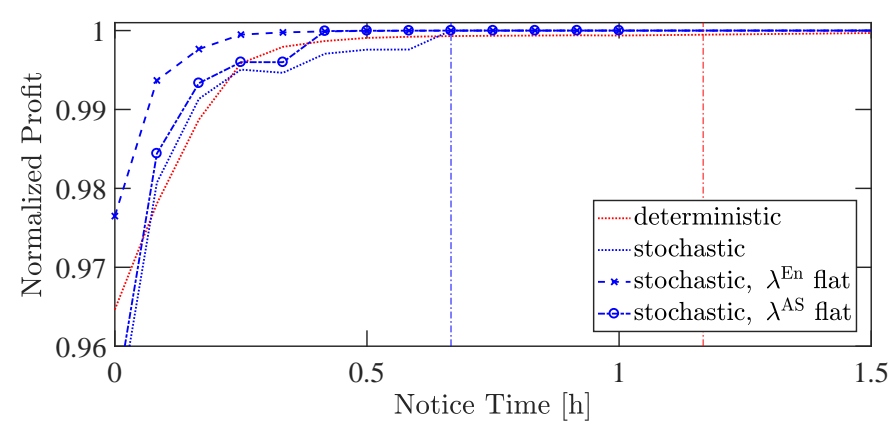

Figure 11. Normalized profits as a function of notice time for a $4 \mathrm{~h}$ planning horizon. flat $=$ constant price

below than the energy price the optimal strategy changes to decrease AS capacity bids in favor of more energy arbitrage. In this case, the total AS capacity can change with notice time; however, this is not realistic given today's market prices.

\section{Conclusions}

In this paper, we developed deterministic and stochastic formulations for an aggregator to optimize aggregate TCL power consumption to arbitrage energy prices and provide ancillary service capacity and investigated the impact of price notice time on the aggregator's profits and flexibility offered to the system. We showed how the stochastic formulation quickly results infeasibility due to the aggregation of the baseline error. In order to avoid this problem, we developed a method to iteratively compensate the baseline error.

The results show that the total flexibility from the TCL aggregation measured as the sum of total AS capacity bids over the planning horizon is not significantly affected by notice time. This result is valid if the AS prices are of the same order of magnitude as the energy prices. The results also show that the aggregator's profits increase as notice time increases up to a point (40 $\mathrm{min}$ to $1 \mathrm{~h} 10 \mathrm{~min}$ ) and then plateau.

We have shown that it may be important to optimize price notice times. One the one hand, system operators interested in encouraging demand side asset participation in energy and ancillary service markets need to ensure aggregators can earn sufficient profits by participating in these markets. To achieve maximum profits the notice time should be set to the point at which profits plateau. On the other hand, since the uncertainties that drive supply/demand mismatch, such as renewable energy generation and load forecasts errors increase over the forecast horizon it is in the interest of the power system operator to hold off on setting prices (and therefore incentives for loads to increase/decrease consumption) until the mismatch is known (i.e., notice time $=0 \mathrm{~min}$ ). Analyzing this trade-off and determining the system-optimal notice time is a subject for future work.

\section{ACKNOWLEDGMENTS}

This research was partially supported by US NSF Grant \#CCF-1442495.

\section{REFERENCES}

[1] J. L. Mathieu, M. Kamgarpour, J. Lygeros, G. Andersson, and D. S Callaway, "Arbitraging intraday wholesale energy market prices with aggregations of thermostatic loads," IEEE Transactions on Power Systems, vol. 30, no. 2, pp. 763-772, 2015.

[2] D. S. Callaway, "Tapping the energy storage potential in electric loads to deliver load following and regulation, with application to wind energy," Energy Conversion and Management, vol. 50, no. 5, pp. 1389-1400, may 2009.

[3] S. Koch, M. Zima, and G. Andersson, "Active Coordination of Thermal Household Appliances for Load Management Purposes," IFAC Proceedings Volumes, vol. 42, no. 9, pp. 149-154, 2009.

[4] S. Kundu, N. Sinitsyn, S. Backhaus, and I. Hiskens, "Modeling and control of thermostatically controlled loads." Power Systems Computation Conference (PSCC), 2011.

[5] J. L. Mathieu, S. Koch, and D. S. Callaway, "State Estimation and Control of Electric Loads to Manage Real-Time Energy Imbalance," IEEE Transactions on Power Systems, vol. 28, no. 1, pp. 430-440, feb 2013.

[6] H. Hao, B. M. Sanandaji, K. Poolla, and T. L. Vincent, "Aggregate Flexibility of Thermostatically Controlled Loads," IEEE Transactions on Power Systems, vol. 30, no. 1, pp. 189-198, jan 2015.

[7] “U.S. Energy Information Administration, annual energy review," 2010. [Online]. Available: https://www.eia.gov/totalenergy/data/annual/

[8] N. Mahdavi, J. H. Braslavsky, and C. Perfumo, "Mapping the Effect of Ambient Temperature on the Power Demand of Populations of Air Conditioners," IEEE Transactions on Smart Grid, pp. 1-1, 2016.

[9] J. Mathieu, M. González Vayá, and G. Andersson, "Uncertainty in the flexibility of aggregations of demand response resources," in Proceedings of the IEEE Industrial Electronics Society Annual Conference, Vienna, Austria, Nov. 2013.

[10] ISO New England Inc., "ISO New England - Pricing Reports," 2017. [Online]. Available: https://www.isone.com/isoexpress/web/reports/pricing?category=Fivemin

[11] California ISO, "Regulation Energy Management Draft Final Proposal," California ISO, Tech. Rep., 2011. [Online]. Available: https://www.caiso.com/Documents/RevisedDraftFinalProposalRegulationEnergyManagement-Jan13_2011.pdf

[12] C. M. Grinstead and J. L. Snell, Introduction to Probability, 2nd ed. American Mathematical Society, 2012. 